\title{
Stressfaktor Ankleiden - so geht es deutlich entspannter
}

\section{Angepasste Kleidung Das An- und Auskleiden von hilfebedürftigen Senioren und} von Menschen mit schweren Krankheiten oder Behinderungen gehört im Pflegealltag zur Routine. Je nach Ausmaß der körperlichen Einschränkungen, stellt diese Tätigkeit einen erheblichen Zeit- und damit auch Stressfaktor für die Pflegekräfte und die Betroffenen dar. Mittlerweile gibt es jedoch - im Alltag viel zu selten verwendete funktionelle und zugleich modische Kleidung für die gehandicapten Menschen. Der folgende Beitrag beschreibt, welche Aspekte bei der Auswahl entsprechender Kleidungsstücke beachtet werden müssen und wie sich durch funktionale, an die jeweilige geistig-körperliche Situation angepasste Kleidung, Zeitnot, Frustration und Stress bei allen Beteiligten vermindern lassen.

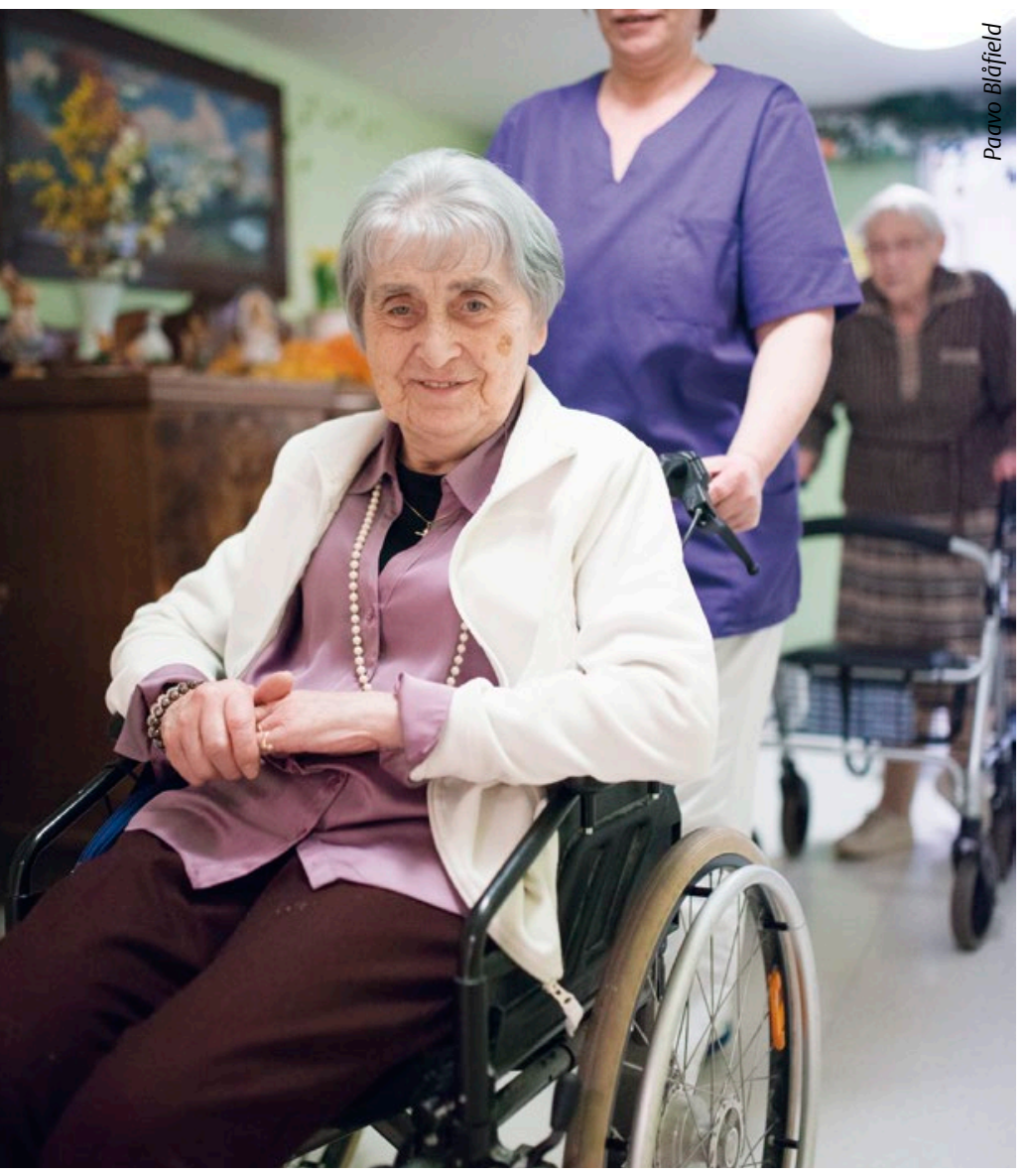

Wer auf Hilfe angewiesen ist, wünscht sich ein komfortables, stressfreies Ankleiden - das freut den Patienten wie auch den assistierenden Helfer (Symbolbild).
- Lucina Zimmermann -

Funktionale Pflegemode

Die an Demenz erkrankte Frau Peters hat sich eingenässt. Das kommt häufig vor, weil sie ihre Inkontinenzvorlage „entsorgt“, sobald sie sich unbeobachtet fühlt. Erneut muss die sie betreuende Pflegekraft, Frau Röder, beim Wechseln der Kleidung helfen. Das blaue Lieblingskleid kommt dabei nicht infrage, weil der Einstieg zu eng ist. Also entscheidet sich Frau Röder für eine Jogginghose, die zwar nicht fraulich und nicht attraktiv ist, aber wenigstens schnell angezogen werden kann. Das Ergebnis ist für beide unbefriedigend, denn normale Kleidung ist auch im Alter ein wichtiges Stück Lebensqualität. ${ }^{1}$

Wie Frau Peters geht es vielen Menschen. Die Zahl der Pflegebedürftigen stieg allein in Deutschland von Dezember 2013 bis Dezember 2015 um 8,9 \% auf 2,86 Millionen Menschen an. Das entspricht in diesem Zeitraum einem Zuwachs von 234.000 pflegebedürftigen Personen - Tendenz weiter steigend. Zudem haben 7,6 Millionen Menschen in Deutschland eine körperliche Schwerbehinderung. ${ }^{2}$ 


\section{Stressfaktor An- und Auskleiden}

Neben der Körperhygiene, der Nutzung von Hilfsmitteln und der Medikamentengabe gehört das zuweilen mehrmalige Anund Auskleiden zur Alltagsroutine, die häufig jedoch als stressreich empfunden wird. Die Gründe dafür sind:

- Durch die körperlichen Einschränkungen wird jedes Ankleiden zu einer zeitraubenden und anstrengenden Prozedur - und damit zu einem Stressfaktor.

- Kleidungsstücke sind in der Regel für Menschen ausgelegt, die über ein gewisses Maß an Beweglichkeit und Feinmotorik verfügen.

Insofern ist diese These wenig gewagt: Durch funktionale, an die jeweilige geistigkörperliche Situation angepasste Kleidung lassen sich Frustration, Stress und Zeitnot vermindern.

\section{Mode und Funktionalität}

Auch wenn - je nach Krankheitsbild und/ oder Grad der Behinderung - existenzielle Probleme des Patienten in den Vordergrund rücken, möchte doch niemand völlig „unmodisch“ gekleidet sein. Nicht nur als Ausdruck unserer Zeit schenkt Mode jedem Einzelnen die Möglichkeit, sich individuell $\mathrm{zu}$ kleiden. ${ }^{3}$ Für schwerkranke und behinderte Menschen kommt also zusätzlich zu den körperlichen Einschränkungen noch der Verlust an identitätsstiftender Mode - denn: Kleidung ist mehr

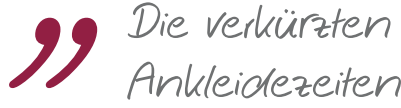
während der Grundpflege und das rückenschonende Arbeiten bei der Versorgung eines bettlägerigen Patienten stellen einen Pluspunkt für die Pflegekrätte dar.

Hajo Kruse, Heimleiter kungen sowie im Bett erleichtern $(\rightarrow$ Abb. 2).

3. Schnitt und Verarbeitung sollen den Patienten unterstützen, damit er sich gut und frei bewegen kann.

4. Hosen sollen - ohne am Bauch zu kneifen - ein bequemes Sitzen ermöglichen.

5. Die Kleidung muss so geschnitten und verarbeitet sein, dass sie im Liegen keine Druckstellen verursacht und nicht zu Dekubiti führt.

6. Kleidung soll Bewegungsabläufe sicher begleiten, Sturz- und Verletzungsrisiken mindern.

7. Funktionelle Kleidung erleichtert den Alltag von Inkontinenzpatienten; Vorlagen passen bequem und lassen sich leichter wechseln.

8. Die Versorgung von Stoma, Shunt und anderen wichtigen Zugängen wird durch spezielle Kleidungsstücke optimiert.

9. Die Kleidung muss einer hohen Beanspruchung standhalten und darf auch durch häufiges Waschen und Trocknen nicht aus der Form geraten. ${ }^{5}$

Eine optimale Pflegemode hat daher ästhetischen, funktionellen und sogar gesundheitserhaltenden Vorgaben zu genügen. ${ }^{6}$

\section{Helfer im} Pflegealltag Eine nicht funktionsgerechte Kleidung führt bei den Pflegenden - und zwar sowohl bei den pflegenden Angehörigen, als auch bei den professionell Pflegenden - zu einem nur schwer als nur eine gut funktionierende Hülle für den Körper., 4,6

Im Bereich der Pflege treten darüber hinaus funktionelle Anforderungen an die Kleidung in den Vordergrund:

1. Gut durchdachte Einstiege für Personen, die die Arme nicht heben können, im Rollstuhl sitzen oder bettlägerig sind ( $\rightarrow$ Abb. 1).

2. Passende Verschlüsse, z.B. aus Klett, die das Anziehen auch für Finger mit Arthritis oder anderen Gelenkerkran-

kalkulierbaren Mehraufwand. Zum einen kann sich der Patient nicht so gut oder überhaupt nicht mehr selbst ankleiden, wenn er mit Knöpfen oder Reißverschlüssen Probleme hat, zum anderen ist das Anziehen, z.B. eines engen Kleidungsstücks, zumeist mit erheblicher Anstrengung verbunden. Die Folgen sind Schmerzen und Stress beim Patienten und Frustration beim Pflegenden.

Im Umkehrschluss heißt das: Durch funktionelle Mode ist ein Patient eher in

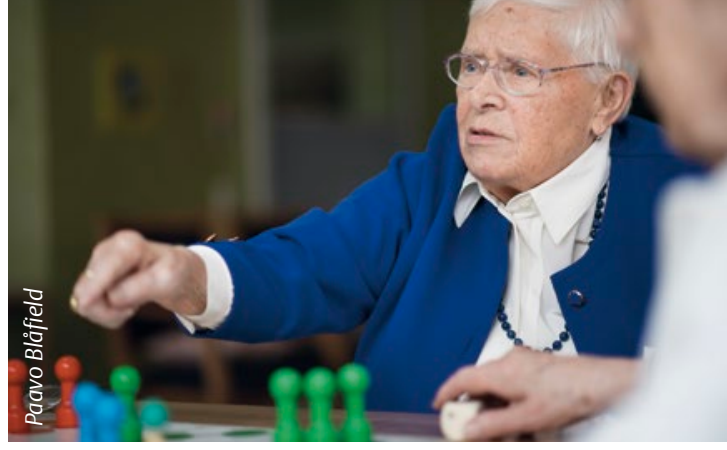

Für die Teilhabe an der Gemeinschaft und an Geselligkeiten spielt die Erhaltung eines guten Aussehens für viele Senioren eine wichtige Rolle. Der Faktor Kleidung trägt hier erheblich zum Wohlbefinden bei (Symbolbild).

der Lage, sich selbst zu helfen. Das erhöht seine Selbstständigkeit und sein Selbstwertgefühl. Eine adäquate Kleidung hilft den Pflegenden, Arbeit zu vermeiden und Zeit zu sparen.

\section{Oberteile}

Pflegemode erleichtert folglich durch ausgeklügelte Verschlüsse und an das jeweilige Krankheitsbild angepasste Schnitte den Pflegealltag. Das wird auch bei der Oberbekleidung deutlich. Im Gegensatz zu herkömmlichen Shirts sind Front-Oberteile hierbei mit Klettverschlüssen oder Magnetschließungen versehen. Dabei sind im Saum Magnetelemente eingearbeitet, sodass z. B. das Öffnen und Schließen eines Kleidungsstücks selbst für arthritische Finger möglich ist ( $\rightarrow$ Abb. 3). Allerdings ist bei Produkten mit Magnetschließungen zu beachten, dass diese z. B. bei Patienten mit Herzschrittmachern kontraindiziert sind.

Für viele Patienten sind Oberteile, die am Rücken vollständig geöffnet werden können, eine gute Option. Das Ankleiden erfolgt von vorn, sodass die Arme ohne Heben oder Senken in die Ärmel gebracht werden können. Auch die Enge eines meist zu kleinen Halsausschnitts fällt weg. Die Oberteile lassen sich mittels zweier überlappender Rückenteile und an den Schultern angebrachten Verschlüssen so schließen, dass der Rücken zu jedem Zeitpunkt vollständig bedeckt ist.

\section{Hosen}

Auch im Bereich der Hosen gibt es mittlerweile Produkte, die das Leben und die Pflege erleichtern, wie das z. B. bei einem Patienten der Fall ist, der durch Kontrakturen extrem bewegungsunfähig ist und beim Anziehen einer normalen Hose mehrmals aufwendig gedreht werden muss.

Klett-Hosen sind für mobile Senioren mit leichten Handicaps geeignet. Das 


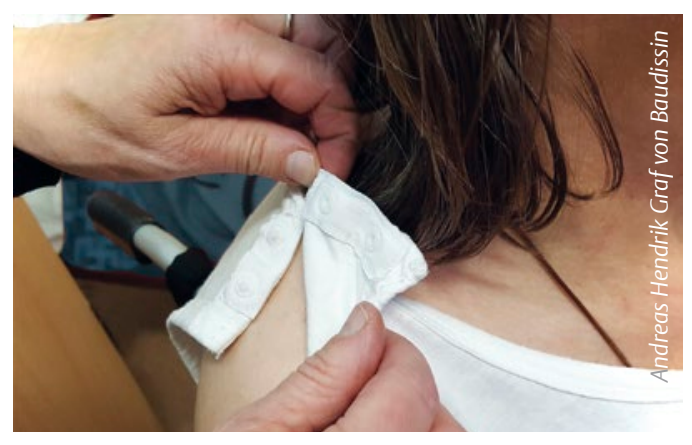

Abb. 1 Das Hemdchen mit Druckknöpfen lässt sich an den Schultern rechts und links öffnen. Es kann angezogen werden, ohne die Arme zu heben oder zu senken.

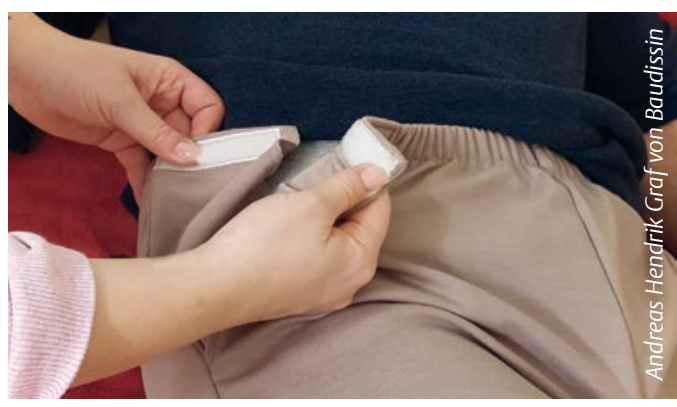

Abb. 2 Eine Schlupfhose mit seitlichen Klettschließungen ist ideal für Patienten, die eine Inkontinenzversorgung brauchen. Gerade in dringenden Momenten lässt sich die Hose schnell öffnen und der Einstieg ist weiter als bei einer herkömmlichen Schlupfhose.

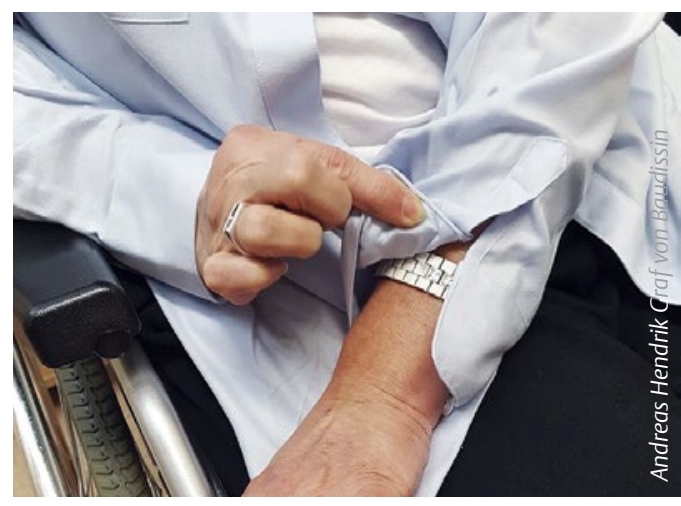

Abb. 3 Eine Bluse mit Magnetverschluss lässt sich auch mit arthritischen Fingern leicht anziehen. Das schwierige Knöpfen entfällt.

Bündchen ist elastisch, jedoch zusätzlich rechts und links durch eine Klettverschlussöffnung ergänzt, sodass der Einstieg in die Hose deutlich vereinfacht ist ( $\rightarrow$ Abb. 2).

Spezielle Pflegehosen für immobile Senioren mit erheblichem Pflegebedarf sind am Bündchen elastisch und lassen sich zusätzlich hinten oder am Bein vollständig

\section{Der Aspekt einer durchdachten Kleidung mit praktischen Funktionen sollte auch zur Erhaltung eines würdevollen} Aussehens beitragen.

Christa Mantel, Pflegedienstleitung

öffnen. Dadurch ist das Ankleiden auch im Liegen möglich.

Zipperhosen mit seitlichen Reißverschlüssen erleichtern den Einstieg und ermöglichen den Komfort, sie an den Beinen selbst leicht zu öffnen und zu schließen.

Rollstuhlfahrerhosen sind am Rücken ergonomisch höher geschnitten, Kniebereiche sind ausgeformt und die Beinlängen für eine sitzende Person angepasst.

Transferhosen sind für Rollstuhlfahrer und Personen mit unsicherem Steh- und Gangbild geeignet. Sie haben am Hosenbein und am Rückenbündchen zusätzlich seitliche Nylongriffe, an denen die Begleitperson ergonomisch dem Rollstuhl angepasst sind und die sich von vorn kleiden lassen und hinten verschlossen werden. Zudem sind wärmende Capes schnell und unkompliziert angezogen.

\section{Hygiene}

In Pflegeeinrichtungen und Krankenhäusern darf bei allen modischen Überlegungen und Funktionalitäten bei der Bekleidung besonders die Hygiene nicht in den Hintergrund treten. Denn dort, wo viele Menschen mit geschwächtem Immunsystem zusammentreffen, kommt es immer wieder zur Verbreitung von Erregern - von der Grippe
9 Besonders bei Kontrakturen und Lähmungen wird das An- und Ausziehen zu einer Herausforderung. Durch das Ankleiden von vorn müssen die betroffenen Arme nickt nach hinten bewegt werden; sie werden einfack in die Ärmel hineingeführt und hinten arn Rücken wird die Kleidung geschlossen. Damit lassen sich Schmerzen vermeiden. Petra Jensen, Kinaesthetics-Trainerin

bei Bedarf oder beim Transfer zugreifen kann, sodass die betroffene Person beim Stehen oder beim Umsetzen vom Rollstuhl auf einen Sessel gesichert werden kann.

\section{Unterwäsche und Nachtwäsche}

Bei der Unter- und Nachtwäsche kommt in der Regel noch der Schamfaktor hinzu. Mittlerweile gibt es jedoch verschiedene Varianten an Nachtwäsche mit leichtem Einstieg, die ein hohes Maß an Intimität wahren und nicht wie ein Krankenhaushemdchen entblößen. Hemdchen, die an den Schultern geöffnet werden können, ganz zu öffnende Unterhosen und spezielle Inkontinenzwäsche erleichtern das Kleiden.

\section{Jacken, Capes und Ponchos}

Auch für den Außenbereich sind für Rollstuhlfahrer praktische Lösungen gefunden worden. Es gibt Jacken, die in ihrem Schnitt über den Norovirus bis hin zu MRSA.7

Das Waschen der Kleidung z.B. von MRSA-Patienten erfolgt mit einem anerkannten, auf seine Wirksamkeit überprüften Wäschedesinfektionsverfahren, um eine weitere Verbreitung des Keims zu unterbinden. ${ }^{8}$ Die Qualität der Kleidung muss diesen Anforderungen genügen und sie muss wieder zügig im Kleiderschrank des Patienten griffbereit liegen, also pflegeleicht und bügelfrei sein.

\section{Krankheitsbilder, Pflegeprobleme -} und Mode

Je nach Krankheit oder Pflegeproblem kann Pflegemode unterstützend wirksam sein, wie die nachfolgenden Beispiele zeigen.

\section{Dekubitus}

In diesem Fall sollten Kleidungsstücke so ausgewählt werden, dass auf harte Näh- 
te und Verschlüsse (vor allem im Sitzund Rückenbereich, am Hinterkopf und an den Fersen) verzichtet wird: Reißverschlüsse, Zipper und Knöpfe dürfen nicht auf sensible Hautstellen drü-

9 Wir sparen durch die speziellen Kleidungsstücke Zeit und auch Kraft. Besonders für Kolleginnen, die aufgrund der körperlichen Anstrengung des Berufs schon selbst Probleme mit der Schulter haben, ist das eine grope Erleichterung.

Jutta Delrieux, Altenpflegerin

cken. Außerdem empfehlen sich weiche und leichte Stoffe sowie nicht zu enge elastische Bündchen an Ärmeln, Hosenbeinen oder in der Taille.

\section{Sturzprophylaxe}

Neben festem Schuhwerk und guten Stoppersocken gilt, dass die Kleidung weder zu lang noch zu weit sein darf, damit ein Hängenbleiben, Hineintreten oder Sich-Verfangen vermieden wird. Für sturzgefährdete Personen bieten sich Hüftschutzgurte und Protektorenhosen mit extra Polsterungen an den gefährdeten Stellen an. Der Nachteil ist, dass einige Patienten, z.B. mit Demenzerkrankung, beim Ankleiden dieser Protektoren nur wenig Geduld aufbringen. Hilfreich sind hier bequeme und großzügige Einstiege in Hosen, die mit Klettverschlüssen unkompliziert geschlossen werden können.

\section{Mobilität fördern und erhalten}

Eine wichtige Voraussetzung für die Teilhabe am sozialen Leben ist die Mobilität des Betroffenen. Da die fehlende und eingeschränkte Mobilität gesundheitli-
Ein Patient mit spastischer Lähmung im Arm hat einen sehr hohen Tonus in der Muskulatur, was das Ankleiden erheblich erschwert. Der gelähmte Arm hingegen ist „einfach nur schwer“; dadurch wird das Ankleiden mit herkömmlicher Kleidung für den Betroffenen aufwendig. Das Schließen der Knöpfe oder eines Reißverschlusses ist einhändig machbar, erfordert aber viel Geduld.

Die passende Kleidung schenkt Motivation und ermöglicht dem Betroffenen, selbstbewusst am sozialen Leben teilzunehmen. Einsamkeit und Rückzug können vermieden werden, wenn es doch nur darum geht, die für die verschiedenen mobilitätseinschränkenden Situationen passende Kleidung auszusuchen.

Bei Harn- oder Stuhlinkontinenz gilt es vor allem, Kleidungsstücke auszuwählen, die sich schnell wechseln lassen. Dazu eignet sich Bekleidung mit Klettverschlüssen, dehnbaren Bündchen sowie seitlichen oder vorn angebrachten Schließungen.

\section{Inkontinenz}

\section{9}

Die Patienten haben unter anderem durch die Physiotherapie wieder gelernt, sich selbst anzukleiden. Mit Balance und Tricks - zumeist sitzend-lernt der Patient, sich in die Kleidung hineinzubewegen. Aber eine Hilfestellung durch einen Dritten ist weiterhin notwendig.

Chris Langston, Physiotherapeut

che Folgen, wie z. B. Dekubitus, Kontrakturen, Sturzgefahr, Obstipation, Thrombose und Pneumonie, haben kann, müssen die Rahmenbedingungen für den mobil eingeschränkten Menschen optimiert werden. ${ }^{9}$

\section{Demenzielle} Erkrankungen Druckknöpfe, Gummizüge und Klettverschlüsse ermöglichen Menschen mit Demenz ein einfacheres Anziehen. Wichtig ist auch die Auswahl der Lieblingsfarben, bekannte Muster und Qualitäten, die schon früher gern getragen wurden. So ist es z.B. wenig sinnvoll, eine Hose in Jeansstoff zu verwenden, wenn die betroffene Person das Tragen von „Nietenhosen“ schon früher abgelehnt hat.
Mehr Lebensqualität für Frau Peters

Auch bei dem Beispiel von Frau Peters war die Anschaffung von spezieller Pflegemode hilfreich. Seit die Hosen einen Klettverschluss haben und die Oberteile nicht über den Kopf gezogen werden müssen, ist das Umziehen nicht mehr mit einem Kraftakt verbunden.

Gleichzeitig fühlt sich Frau Peters, die trotz ihrer Demenz großen Wert auf die äußere Erscheinung legt, sichtlich wohl in ihrer neuen Kleidung.

\section{Literatur}

1 Beispiel aus: Zimmermann L. Pflegemode. Wie das Ankleiden leichter wird, Hamburg 2017, S. 10. Viele Aussagen dieses Artikels stammen aus diesem Buch, Windsor Verlag 2017

2 Statistisches Bundesamt. Pflegestatistik 2015. Pflege im Rahmen der Pflegeversicherung. Deutschlandergebnisse. Wiesbaden, 2017; Statistisches Bundesamt: Pressemitteilung vom 24.10.2016 - 381/16, Wiesbaden 2016

3 Justo G. Kleidung als symbolische Selbstinszenierung. München, Grin Verlag, 2008; vgl. darin Kap. 3. Die Selbstinszenierung durch Kleidung, S. $46 \mathrm{ff}$,

4 Vgl. dazu u. a. die Anmerkungen in http://www. pflegewiki.de/wiki/Kleidung; Zugriff: 16.12.2017

5 Zimmermann, ebd., S. $22 f$

6 Der Begriff „Pflegemode“ umfasst die Kleidungsstücke, die das Ankleiden auch dann noch angenehm und leicht gestalten, wenn besondere Umstände hinzukommen.

7 Vgl. http://www.mrsa-net.nl/de/oeffentlichkeit/ mrsa-allgemein/was-ist-mrsa/274-was-ist-mrsa; Zugriff: 14.12.2017.

8 Bundesgesundheitsblatt - Gesundheitsforschung - Gesundheitsschutz 6 - 2014; https:// www.springermedizin.de/empfehlungen-zurpraevention-und-kontrolle-von-methicillin-resis/8013136; Zugriff: 16.12.2017.

9 http://www.ksfh.de/files/Weiterbildung/Umsetzung_Expertenstandards_Reuther_Kutschke.pdf; Zugriff: 16.12.2017.

\section{Autorin}

\section{Lucina Zimmermann}

Betriebswirtin in der Seniorenwirtschaft, seit 2004 im Bereich der Pflege tätig,

Nominierung für den schleswig-

holsteinischen Altenpflegepreis „Pflege in

der Kunst" (2011) und für den Alten-

pflegepreis des Vincentz-Verlags zum

Thema „Kleiden bei Pflegebedürftigkeit“

(2015), Autorin des Buches „Pflegemode -

Wie das Ankleiden leichter wird“.

lucina.zimmermann@gmx.de

\section{Bibliografie}

DOI 10.1055/s-0044-101763

GGP 2018; 2: 66-69

(c) Georg Thieme Verlag KC

Stuttgart · New York · ISSN 2511-7548 